Artigo original

\title{
Ginástica hipopressiva como recurso proprioceptivo para os músculos do assoalho pélvico de mulheres incontinentes
}

\section{Hypopressive gymnastics as resource for perineal proprioception in women with urinary incontinence}

\author{
Thaís F Costa, Ft.*, Ana Paula M Resende, Ft., D.Sc.**, Maura R Seleme, D.Sc.**, Liliana Stüpp, D.Sc.**, \\ Rodrigo A. Castro, D.Sc. ${ }^{* * *}$, Bary Berghmans, D.Sc. ${ }^{* * * *}$, Marair GF Sartori, D.Sc. ${ }^{* * * * *}$
}

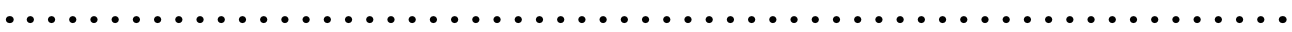

*Fisioterapeuta, Mestranda em Ciências pelo Departamento de Ginecologia da Universidade Federal de São Paulo/ Escola Paulista de Medicina, **Fisioterapeuta, Doutora em Ciências pelo Departamento de Ginecologia da Universidade Federal de Säo Paulol Escola Paulista de Medicina, ***Fisioterapeuta, Doutora em Ciências Sociais pela Universidade Federal do Rio de Janeiro, ****Professor Adjunto do Departamento de Ginecologia da Universidade Federal de São Paulo/ Escola Paulista de Medicina, *****PhD em Epidemiologia Clínica, Maastricht, Holanda, ******Professora Associada Livre Docente do Departamento de Ginecologia da Universidade Federal de Sáo Paulo/ Escola Paulista de Medicina

\section{Resumo}

Introdução: Está amplamente descrito na literatura que as mulheres com e sem disfunção do assoalho pélvico apresentam grande dificuldade em realizar a contração adequada dos músculos perineais. A Ginástica Hipopressiva poderia facilitar o entendimento da correta contraçáo uma vez que supostamente promove contraçáo reflexa dos músculos do assoalho pélvico (MAP) durante sua realização. Objetivo: Avaliar a função dos MAP antes e após protocolo de exercícios hipopressivos para melhora da propriocepção perineal em mulheres com incontinência urinária de esforço (IUE). Métodos: Foram incluídas 14 mulheres com IUE que se submeteram a protocolo de três sessóes individuais, com conteúdo padronizado, que incluiu o aprendizado dos exercícios hipopressivos em etapas. Após este período de aprendizado, as pacientes se submeteram a 12 semanas de exercícios domiciliares, com sessôes mensais com o fisioterapeuta. A cada sessão, as pacientes apresentavam diário de exercícios preenchido para mensuração da aderência ao tratamento. A função dos MAP foi mensurada antes e após o tratamento por meio de palpaçáo bidigital, utilizando-se a escada de Oxford, o endurance muscular e o número de contraçóes rápidas. Resultados: Houve melhora em todos os parâmetros de função muscular avaliados: Oxford $(\mathrm{p}=0,0005)$; endurance $(\mathrm{p}=0,0001)$ e número de contraçôes rápidas $(\mathrm{p}<0,0001)$. Conclusão: Houve aumento da função muscular do assoalho pélvico após prática de exercícios hipopressivos para melhora da propriocepção perineal.

Palavras-chave: incontinência urinária de esforço, propriocepção perineal.

\begin{abstract}
Introduction: It is widely reported in the literature that women with and without pelvic floor dysfunction have great difficulty in performing adequate contraction of the perineal muscles. Hypopressive Gymnastics could facilitate the correct understanding of contraction since that supposedly promotes reflex contraction of the pelvic floor muscles (PFM) during its implementation. Objective: To evaluate the function of PFM before and after hypopressive exercise protocol to improve perineal proprioception in women with stress urinary incontinence (SUI). Methods: Were included 14 women with SUI who underwent a protocol of 3 individual sessions with standardized content, which included learning of the hypopressive exercises in stages. After this learning period, patients underwent 12 weeks of home exercise, with monthly sessions with the physiotherapist. At each session, the patients presented daily exercises journal to measure adherence. The role of PFM was measured before and after treatment by bidigital palpation, using the Oxford scale, muscular endurance and the number of rapid contractions. Results: Was observed improvement in all evaluated parameters of muscle function: Oxford $(\mathrm{p}=0.0005)$, endurance $(\mathrm{p}=0.0001)$ and number of rapid contractions $(\mathrm{p}<0.0001)$. Conclusion: Was observed increased pelvic floor muscle function after hypopressive exercise for improving perineal proprioception.
\end{abstract}

Key-words: perineal proprioception, stress urinary incontinence. 


\section{Introdução}

De acordo com a International Continence Society (ICS), a função adequada dos músculos do assoalho pélvico (MAP) é definida como habilidade em realizar contração voluntária forte e presença de contração involuntária, que resultam em fechamento circular da vagina, uretra e ânus em movimento cranioventral do períneo e elevação dos órgáos pélvicos [1]. Essa contração é importante para prevenir perda involuntária de urina ou fezes [1].

Diversos autores evidenciam a dificuldade que as mulheres apresentam em contrair adequadamente os MAP [2-4]. Em mulheres sem disfunção do assoalho pélvico, foi descrito que $37,2 \%$ não possuem habilidade em contrair corretamente mesmo após uma sessão com orientaçôes, e ainda que $25 \%$ dessas mulheres realizam o movimento oposto à contraçáo correta, a manobra de Valsalva [2]. Em estudo mais recente foi relatado $44,9 \%$ de inabilidade de contraçáo dos MAP em mulheres sem disfunçóes [3]. Já em mulheres incontinentes, a dificuldade é ainda maior, e abrange 65,5\% dessas [4].

A Ginástica Hipopressiva foi proposta como alternativa para o tratamento das disfunções do assoalho pélvico e, dentre suas indicaçôes, encontra-se a melhora da propriocepçáo dos MAP [5,6].

Esses exercícios são praticados em três fases: 1) inspiração diafragmática lenta e profunda, 2) expiração completa e, 3) aspiração diafragmática, em que ocorre progressiva contração dos músculos abdominais profundos, intercostais e elevação das cúpulas diafragmáticas [5,7].

A aspiração diafragmática supostamente promove pressão negativa na cavidade abdominal e, reflexamente, ativa os MAP por meio de tração da fáscia abdominal, que é conectada à fáscia endopélvica [5,7]. Assim, essa ativação reflexa dos MAP facilitaria o aprendizado da correta contração [5].

Entanto, apesar dessas indicaçôes existem poucas evidências de que a prática dos exercícios hipopressivos possa efetivamente auxiliar o aprendizado da correta contração dos MAP. O objetivo do presente estudo foi avaliar os efeitos de um protocolo de ginástica hipopressiva no aprendizado da contração dos MAP em mulheres com incontinência urinária de esforço (IUE).

\section{Material e métodos}

No período de fevereiro a dezembro de 2009 , foram selecionadas 14 pacientes do Ambulatório de Uroginecologia e Cirurgia Vaginal da Universidade Federal de São Paulo (UNIFESP), São Paulo, Brasil. As participantes desse estudo piloto foram recrutadas por contato pessoal.

Os critérios de inclusão adotados foram a presença de IUE, sem defeito esfincteriano, confirmada por estudo urodinâmico. Como critérios de exclusão, adotaram-se a presença de doenças degenerativas, diabetes e hipertensão descompensados e realização prévia de tratamento fisioterapêutico para IUE.
As pacientes que concordaram em participar do estudo, assinaram o termo de consentimento livre e esclarecido, aprovado pelo Comitê de Ética em Pesquisa da UNIFESP por meio do protocolo $n^{\circ} 1978 / 07$.

Para o diagnóstico da IUE, as mulheres passaram pelos procedimentos médicos de rotina (anamnese, exame físico geral e exame ginecológico).

Uma vez incluída no estudo, a paciente foi avaliada por fisioterapeuta especializado em uroginecologia, que mensurou a função muscular por meio de palpação bidigital, utilizando-se a escala de Oxford, o teste do tempo de manutenção da contração em segundos, também chamado de endurance muscular e o número de contraçóes rápidas, também chamado de fast. Referida avaliação foi adaptada do método Perfect, descrito por Laycock et al. [8], amplamente utilizado na literatura.

Antes da avaliação, a paciente recebeu orientaçóes sobre a localização e função dos MAP e os procedimentos de avaliação foram explicados em detalhes.

Para a avaliação fisioterapêutica, as pacientes foram orientadas a esvaziar a bexiga e em seguida foram colocadas em posiçáo de litotomia. Foi respeitado um tempo de repouso três vezes maior que o tempo de contraçáo e em seguida foi solicitada a contração mantida pelo máximo de tempo que a paciente conseguisse.

O protocolo de propriocepção com ginástica hipopressiva foi constituído de 3 sessóes individuais para consciência e aprendizado dos exercícios, uma vez por semana. Essas sessóes foram conduzidas por um segundo fisioterapeuta.

A primeira sessão consistiu de esclarecimentos sobre a localização e função dos MAP e dos músculos abdominais. A seguir, as pacientes observaram e localizaram o próprio assoalho pélvico com o auxílio de espelho. Em seguida, foram instruídas a realizar a contraçáo isolada desses músculos e, para a facilitação do aprendizado. A Ginástica Hipopressiva foi ensinada em etapas. Inicialmente, as pacientes foram instruídas quanto à respiração diafragmática. Foram incentivadas a inspirar lenta e profundamente pelo nariz e expirar pela boca o máximo de ar que conseguissem. Ao perfazer esse movimento de expiração, os músculos transversos abdominais eram ativados. Em seguida, foram usados comandos verbais como "abaixe as costelas" e "aproxime o abdômen das costas" para intensificar a contração dessa musculatura. Por fim, foram instruídas com relação à terceira fase dos exercícios hipopressivos, compreendida por aspiração diafragmática. Nessa manobra ocorre associação entre o fechamento da glote e o movimento de sucção abdominal, em sentido posterior e superior, que resulta em pressão negativa dentro da cavidade abdominal e ativação reflexa dos músculos transversos abdominais e músculos do assoalho pélvico, segundo Seleme et al. [5]. Acredita-se que essa manobra poderia facilitar o aprendizado da contração correta dos MAP.

$\mathrm{Na}$ ultima sessão, as pacientes foram ensinadas a contrair voluntariamente os MAP durante a fase de aspiração diafragmática. Embora houvesse ativação reflexa desses músculos, a 
contração voluntária foi incentivada. Os exercícios foram treinados nas posições deitada e em pé. O tempo de manutenção da aspiração diafragmática associada a contração perineal foi preconizado de acordo com a avaliação inicial dos músculos do assoalho pélvico, individual para cada paciente.

Após o término do período de aprendizado, as mulheres foram encaminhadas para a realização de exercícios domiciliares. Foram preconizadas de 4 a 6 repetiçóes do exercício na posição deitada e de 4 a 6 repetiçôes na posição de pé 2 vezes ao dia, perfazendo, no total, 16 a 24 repetiçôes diárias.

Durante este período de realização de exercícios domiciliares, as pacientes preencheram diário de exercícios e receberam contato telefônico quinzenal pelo fisioterapeuta, para esclarecimento de possíveis dúvidas e manutençáo do incentivo. Além disso, as pacientes receberam uma cartilha de exercícios, detalhando cada etapa, para facilitar o aprendizado.

Ao final de um mês as pacientes retornaram para consulta com o fisioterapeuta. O protocolo de Ginástica Hipopressiva era refeito e o período de manutenção da contração era intensificado, seguindo ritmo natural de progressão do treinamento, com o objetivo de maior recrutamento dos músculos envolvidos no fortalecimento e de não permitir a adaptação do músculo [8]. Esse protocolo foi seguido por três meses (12 semanas). Ao final desse período, as pacientes foram reavaliadas.

No que se refere à análise estatística, os dados foram analisados por meio do software especializado SPSS 13.0. Inicialmente realizou-se estatística descritiva (frequência, média e desvio padrão), posteriormente, analítica, utilizando-se os testes t de Student, para avaliar as diferenças entre as variáveis quantitativas paramétricas, e Qui-quadrado, para as variáveis ordinais. O intervalo de confiança utilizado foi de $95 \%$, e os valores de $p<0,05$ foram considerados significativos, assinalando-se com asterisco.

\section{Resultados}

Das 14 mulheres selecionadas para o estudo, 12 concluíram o tratamento. A média de idade das pacientes foi de 57,6 $( \pm 8)$ anos. O índice de massa corpórea (IMC) médio foi de $27,7( \pm 6,5) \mathrm{kg} / \mathrm{m}^{2}$. O número de gestaçôes foi de 3,5 $( \pm 1,6)$ e o de partos vaginais foi $2,5( \pm 1,8)$.

No que se refere à avaliação dos MAP, os resultados encontrados estão demonstrados na Tabela I.

Tabela I - Avaliação dos músculos do assoalho pélvico, mensurada por meio de ultrassonografia bidimensional e palpação bidigital, no início e ao final do tratamento.

\begin{tabular}{lccc}
\hline \multicolumn{1}{c}{ Variável } & Inicial & Final & p valor \\
\hline Oxford & $1,6( \pm 0,6)$ & $2,8( \pm 0,5)$ & $\mathrm{P}=0,0005^{*}$ \\
Endurance (seg) & $2,7( \pm 1,8)$ & $5,3( \pm 2,1)$ & $\mathrm{P}=0,0001^{*}$ \\
$\mathrm{~N}^{\circ}$ de contrações & $3,4( \pm 1,3)$ & $7,3( \pm 1,9)$ & $\mathrm{P}<0,0001^{*}$ \\
rápidas (fast) & & &
\end{tabular}

"Valores obtidos por meio do Teste t de Student.
No que diz respeito às variáveis mensuradas pelo diário de exercícios, no primeiro mês de tratamento, as pacientes relataram que praticaram os exercícios em média 27,5 dias. No segundo mês a média foi de 27 dias e no terceiro mês de tratamento, as pacientes realizaram os exercícios em média 29 dias.

No que se refere à perda urinária, observou-se que no início do tratamento, todas as pacientes $(n=12)$ perdiam urina. Ao final do tratamento, quatro pacientes relatavam não perder mais urina e 7 pacientes relataram melhora significativa. Seis pacientes no início do tratamento perdiam urina em jato que molhava a roupa e causava constrangimento, e, ao final, somente uma.

\section{Discussão}

Está amplamente descrito na literatura que o treinamento específico para os MAP representam o padrão ouro para o tratamento da incontinência urinária de esforço [10] e, recentemente, seu valor foi reconhecido também no tratamento da bexiga hiperativa e do prolapso genital [11].

Entretanto, para que o treinamento específico seja praticado, é necessário garantir a correta contração dos MAP, pois, não surtiria efeito realizar inúmeras séries de exercícios, incluindo contraçóes rápidas e lentas se o movimento realizado no treinamento não estivesse correto.

Em 1952, Arnold Kegel descreveu a correta contração dos MAP, que envolve dois componentes: fechamento ao redor dos orifícios pélvicos e movimento de elevação cranial dos órgãos. Recentemente, inúmeros autores descreveram pesquisas avaliando a capacidade de contração correta dos MAP de mulheres com e sem disfunção do assoalho pélvico $[2-4,13]$. Outros autores afirmam que pesquisas na área de consciência perineal e aprendizado da correta contração deveriam ser prioridade dos pesquisadores na área do assoalho pélvico [14]. Este estudo piloto objetivou avaliar o efeito de um programa de Ginástica Hipopressiva no aprendizado da correta contração dos MAP.

A função dos MAP no presente estudo foi avaliada por meio de palpação bidigital, utilizando-se a escala de Oxford, bem como o endurance muscular e o número de contrações rápidas derivados da escala Perfect [8]. Segundo Bo e Morkved [14], a palpação bidigital é um dos principais métodos de avaliação utilizados pelos fisioterapeutas e, observa-se que mesmo nos dias de hoje, em que há tantos outros métodos tecnológicos de avaliação (ultrassom, ressonância magnética), a palpação ainda é um dos métodos de avaliação mais utilizados.

Pacientes que possuem adequada contração dos MAP, devem apresentar valores acima de 3 na escala Oxford, sem recrutar outros grupos musculares como adutores, abdominais e glúteos [15]. Em nosso estudo, a função muscular do assoalho pélvico antes do tratamento, mensurada pela escala de Oxford, foi de 1,6 $( \pm 0,6)$, considerada funçáo muscular débil. Entretanto, após período de treinamento envolvendo 
ginástica hipopressiva para o aprendizado da correta contração, observou-se melhora da função muscular, cujo valor foi de $2,8( \pm 0,5)$, aproximando-se da funçáo considerada ideal.

$\mathrm{O}$ endurance muscular aumentou de $2,7( \pm 1,8)$ segundos para 5,3 $( \pm 2,1)$ segundos $(\mathrm{p}=0,0001)$, e o número de contraçôes rápidas aumentou de 3,4 $( \pm 1,3)$ para 7,3 $( \pm 1,9)(\mathrm{p}$ $<0,0001)$. As pacientes foram orientadas a ficar em aspiração diafragmática, ou seja, manter a contração, por maior tempo possível, e tal fato pode ter resultado na melhora significativa do tempo de manutenção da contração.

No que diz respeito à técnica hipopressiva, há carência de estudos que realmente evidenciem o que ocorre com o músculo diafragma e os MAP. Os autores que preconizam a utilização dessa técnica, afirmam que há elevação das cúpulas diafragmáticas e dos MAP ocasionados pela diminuição da pressão dentro da cavidade abdominal durante a manobra de aspiração diafragmática. Recentemente, Talasz et al. [16] evidenciaram íntima correlação entre os movimentos das cúpulas diafragmáticas e do assoalho pélvico por meio de ressonância magnética. Seus resultados demonstraram que quando o diafragma se desloca para baixo, durante a inspiração, o assoalho pélvico igualmente se desloca para baixo e que na expiração ocorre o contrário, as cúpulas diafragmáticas se elevam porque os pulmóes se esvaziam e o assoalho pélvico se eleva na mesma proporção. Estes resultados estão em acordo com as afirmaçôes dos autores que preconizam os exercícios hipopressivos, no que se refere ao movimento sincrônico do diafragma respiratório e do assoalho pélvico.

No presente estudo a aderência foi mensurada por meio de diários de exercícios. No primeiro mês as pacientes praticaram os exercícios em média 27,5 dias, no segundo mês, 27 dias e no ultimo mês as pacientes relaram praticar os exercícios em média 29 dias. Esses dados demonstram elevada aderência ao tratamento.

Embora os exercícios para o assoalho pélvico não sejam invasivos, não apresentem efeitos colaterais e sejam de baixo custo, fatores como baixa aderência da paciente ao tratamento, baixa motivação da paciente e falta de compreensão da terapia podem interferir nos resultados dessa abordagem terapêutica sendo fatores limitantes para esse tipo de abordagem [17]. Este fato não ocorreu no presente estudo, onde a aderência ao tratamento foi elevada.

A literatura aponta como fatores determinantes para o sucesso da terapia a compreensão dos exercícios e o conhecimento do próprio corpo. Por outro lado os fatores negativos seriam a não assimilação da orientação que está sendo oferecida e a não incorporação dos exercícios no dia a dia. Quando inadequado, o treinamento pode gerar resultados insatisfatórios e frustração [17].

No presente estudo as pacientes foram instruídas acerca da localização e função da pelve e dos MAP, fato que as incentivou ao conhecimento do próprio corpo. Além disso, a Ginástica Hipopressiva pode ter sido bem aceita pelas pacientes deste estudo porque trabalha o individuo de forma global e em diversas posturas além de incorporar a respiração e a postura em sua prática.

Todavia, faltou-nos comparar os exercícios hipopressivos com outras técnicas de consciência perineal descritas na literatura. Portanto, sugestóes para novos estudos incluindo outras técnicas de consciência e outras técnicas de avaliação do assoalho pélvico tais como atividade elétrica e volume muscular devem ser consideradas para os pesquisadores dessa área.

\section{Conclusão}

A Ginástica Hipopressiva apresentou resultados positivos na melhora da propriocepção perineal, observados pela melhora da função muscular. Houve elevada aderência das pacientes ao tratamento.

\section{Referências}

1. Messelink B, Benson T, Berghmans B et al. Standardisation of terminology of pelvic floor muscle function and dysfunction: report from the pelvic floor clinical assessment group of the international continence society. Neurourol Urodyn 2005;24:374-80.

2. Bo K, Larsen S, Oseid $S$ et al. Knowledge about and ability to correct pelvic floor muscle exercises in women with urinary stress incontinence. Neurourol Urodyn 1998;7:261-2.

3. Talasz H, Himmer-Perschak G, Marth E et al. Evaluation of pelvic floor muscle function in a random group of adult women in Austria. Int Urogynecol J 2008;19:131-5.

4. Talasz H, Gosch M, Enzelsberger H, Rhomberg HP. Geriatrische Patientinner mit harninkontinenz-symptomen und ihre kontrolle über den beckenboden. Z Gerontol Geriatr 2005;38:424-30.

5. Seleme M R, Bertotto A, Ribeiro VW. Exercícios hipopressivos. In: Palma P. Urofisioterapia: Aplicaçóes clínicas das técnicas fisioterapêuticas nas disfunções miccionais e do assoalho pélvico. Personal link 2009;295-307.

6. Berghmans B et al. Pelvic floor rehabilitation. In: Staskin DR, ed. Atlas of bladder disease. London: Springer; 2010.

7. Caufriez M. Gymnastique abdominale hypopressive. Bruxelles; 1997. p.8-35.

8. Laycock J, Whelan MM, Dumoulin C. Patient assessment. In: Haslam J, Laycock J, eds. Therapeutic management of incontinence and pelvic pain. 2 ed. London: Springer; 2008. p.57-66.

9. Bo K, Ascheoug A. Pelvic floor and exercise science: strength training In: Bo K, Berghmans B, Morkved S, Van Kampen $\mathrm{M}$, eds. Evidence-based physical therapy for the pelvic floor. Elsevier; 2007. p.119-32.

10. Hay-Smith EJC, Dumoulin C. Pelvic floor muscle training versus no treatment, or inactive control treatments, for urinary incontinence in women. Cochrane Database Syst Rev 2010;1.

11. Abrams P, Andersson L, Birder L, Brubaker L, Cardozo C, Chapple A, Cottenden W, Davila D et al. Fourth international consultation on incontinence recommendations of the international scientific committee: evaluation and treatment of urinary incontinence, pelvic organ prolapse and fecal incontinence. Neurourol Urodyn 2010;29:213-40.

12. Kegel AH. Stress incontinence and genital relaxation. Clinical Symposia 1952;4(2):35-51. 
13. Bump R, Hurt WG, Fantl J et al. Assessment of Kegel exercise performance after brief verbal instruction. Am J Obstetr Gynecol 1991;165:322-9.

14. Bo K, Morkved S. Motor learning. In: Bo K, Berghmans B, Morkved S, Van Kampen M. Evidence-based physical therapy for the pelvic floor. Elsevier; 2007. p.113-9.

15. Moen MD, Noone MB, Vassallo BJ, Elser DM. Pelvic floor muscle function in women presenting with pelvic floor disorders. Int Urogynecol J 2009; 20:843-6.
16. Talasz H, Kremser C, Kofler M, Kalchschmid E, Lechleitner M, Rudisch A. Phase-locked parallel movement of diaphragm and pelvic floor during breathing and coughing - a dynamic MRI investigation in healthy females. Int Urogynecol J 2011;22:61-8.

17. Felicíssimo MF, Carneiro MF, Souza ELBL et al. Fatores limitadores à reabilitação da musculatura do assoalho pélvico em pacientes com incontinência urinária de esforço. Acta Fisiátrica 2007;14(4):233-6. 